\title{
Single stage substitution urethroplasty using buccal mucosa in the management of stricture urethra in balanitis xerotica obliterans (BXO)
}

\author{
Manne $\mathbf{V}^{1}$, Kulkarni $\mathbf{P}^{2}$ \\ ${ }^{1}$ Dr Venu Manne, Consultant, Department of Urology, Narayana Medical College and Hospital, Nellore, Andhra \\ Pradesh, India, ${ }^{2}$ Dr Prashant Kulkarni, Consultant, Department of Urology, Narayana Hrudayalaya, Bangulur, India.
}

Address for Correspondence: Dr Venu Manne, Consultant, Department of Urology, Narayana Medical College and Hospital, Nellore, Andhra Pradesh. Address- H. No.11-8-89, Lenin Nagar, Khammam,Telangana, India. E- mail id:venumanne1@gmail.com

\begin{abstract}
Introduction: Urethral stricture is "narrowing of the caliber of the urethra caused by the presence of a scar consequent to infection, inflammation or injury". Urethral strictures are difficult to manage. BXO is more resistant to all types of treatment and recurrence is the most common complication. Buccal mucosa dorsal onlay graft urethroplasty is one of the surgical treatment modality for treating BXO related strictures. Objective and Methods: This study is to assess the outcome of stricture urethra in BXO cases treated by single stage buccal mucosa substitution urethroplasty. A Prospective study, total 35 patients underwent surgery for stricture urethra with BXO. Results: 33 patients underwent single stage dorsal onlay buccal mucosa substitution urethroplasty and 2 patients underwent augmented roof strip buccal mucosa urethroplasty Pre and post operative uroflowmetry done for all patients, a significant increase in post-operative uroflowmetry rates observed (P value is less than $\mathbf{0 . 0 0 0 1}$.). Patients were followed up post-operatively at 3, 6, 9, 12 and 36 months intervals out of 35 patients 6 patients had the recurrence. Out of 6 patients, 3 patients underwent one single procedure for recurrent stricture. the success rate is $91.42 \%$. (mean followup period 36.2 months). Conclusion: Buccal mucosa dorsal onlay urethroplasty a good surgical option for BXO patients with less recurrence rate.
\end{abstract}

Key words- Stricture urethra, Buccal mucosa urethroplasty, Balanitis xerotica obliterans.

\section{Introduction}

Urethral stricture is "narrowing of the caliber of urethra" caused by the presence of a scar consequent to infection, inflammation or injury". Urethral strictures are difficult to manage. Susruta described the management of urethral stricture in 300 BC [1]. Some treatment modalities for urethral strictures are fraught with high patient morbidity and stricture recurrence rates. However, an extremely useful tool in the armamentarium of the Reconstructive Urologist is buccal mucosa urethroplasty.

Amongst the various aetiologies for the development of stricture urethra the most common being traumatic and iatrogenic [2]. Other factors like inflammatory, congenital, malignant and balanitis xerotica obliterans

Manuscript received $10^{\text {th }}$ August 2016

Reviewed: 24 ${ }^{\text {th }}$ August 2016

Author Corrected: $4^{\text {th }}$ September 2016

Accepted for Publication $15^{\text {th }}$ September 2016
(BXO) - lichen sclerosis et atrophicus [LS] also contribute. Balanitis Xerotic obliterans or Penile Lichen Sclerosus is a dermatological condition affecting the genitalia and associated with chronic, progressive, sclerosing inflammatory dermatosis of unclear aetiology [3].

Depending on location, size and type various types of surgical treatments are available for stricture urethra. BXO is more resistant to all types of treatment and recurrence is the most common complication. The first choice for graft material is currently buccal mucosa, which can be used for either 1- or 2-stage urethroplasty [4].

Buccal mucosa dorsal onlay graft urethroplasty is one of the surgical treatment modality for treating BXO related urethral strictures. 


\section{Aim}

This study is to assess the outcome of stricture urethra in BXO cases treated by single stage buccal mucosa substitution urethroplasty.

\section{Materials and Methods}

Study type and place of study- A prospective analysis was carried out, at our institute, Puttaparthi, Anantapur, Andhra Pradesh from 2010 to 2014.

Sample size and evaluation- A total of 35 patients presenting with urethral strictures with suspicious or clinically diagnosed as BXO who were undergone single stage substitution buccal mucosa urethroplasty are included in the study.

On admission patients were clinically evaluated and by using retrograde urethrogram or Sonourethrogram, selectively to know the exact site and length of the stricture. Oral hygiene, preparation were ensured prior to the surgery.
All patients were submitted for urethral mucosa biopsy at stricture site intra operatively for histological confirmation of BXO.

\section{Inclusion criteria}

1. All cases selected are clinically proven cases of BXO.

2. All BXO cases which are undergoing buccal mucosa urethroplasty ONLY, irrespective of stricture length.

3. Prior minimally invasive procedures like Visual Internal Urethrotomy, dilatations for stricture urethra are also considered.

Exclusion criteria- Patients who underwent prior open urethral stricture surgeries were excluded form study.

Success:- Success is defined as the one who voids with a good stream of urine post operatively with or without an auxiliary Procedure (dilatation / Visual Internal Urethrotomy), not more than once.

\section{Results and Analysis}

Total 35 patients with age distribution ranging from 18 yrs to 52 yrs with the mean age of 36 yrs, with the maximum incidence in 3rd and 4th decades.

Out of 35 patients 26 patients presented with lower urinary tract symptoms like poor urinary stream, dysuria, frequency and feel of incomplete voiding and 8 patients had the history of previous interventions like meatoplasty, dilatation of urethra and Visual Internal Urethrotomy.

Serum creatinine levels in this study found to be ranging from $0.9 \mathrm{mg} \%$ to $2.6 \mathrm{mg} \%$ with an average of $1.4 \mathrm{mg} \%$ Stricture length ranges from $3 \mathrm{~cm}$ to $14 \mathrm{cms}$ with the mean length of $7.6 \mathrm{cms}$. 10 patients were having pan anterior urethral stricture, 16 patients with bulbar urethral involvement and 9 patients are having bulbar and proximal penile urethral involvement.

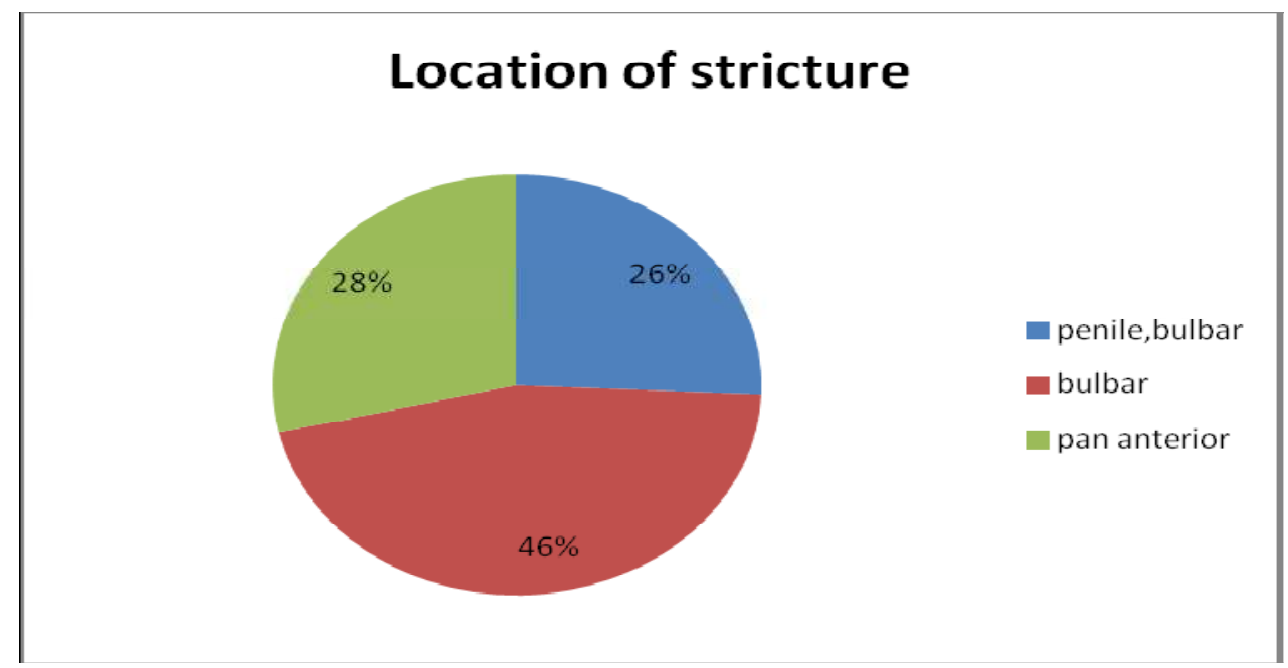

Fig-1: Stricture location 
All patients underwent uroflowmetry study before and after surgery, with pre operative uroflowmetry readings ranging from $4 \mathrm{ml} / \mathrm{sec}$ to $14 \mathrm{ml} / \mathrm{sec}$ with the mean flow of $9 \mathrm{ml} / \mathrm{sec}$. Post-operatively patients underwent uroflowmetry study ranging from $15 \mathrm{ml} / \mathrm{sec}$ to $24 \mathrm{ml} / \mathrm{sec}$ with an average of $19.17 \mathrm{ml} / \mathrm{sec}$.

On statistical analysis:- (comparison of pre and post-operative uroflowmetry values)

Confidence interval: The mean of Group One minus Group Two equals $-10.2495 \%$ confidence interval of this difference: From -11.40 to -9.07 .

Intermediate values used in calculations: $\mathrm{t}=17.9179 \mathrm{df}=33$ standard error of difference $=0.571$

The paired t test $\mathrm{P}$ value is less than 0.0001. By conventional criteria, this difference is considered to be statistically significant.

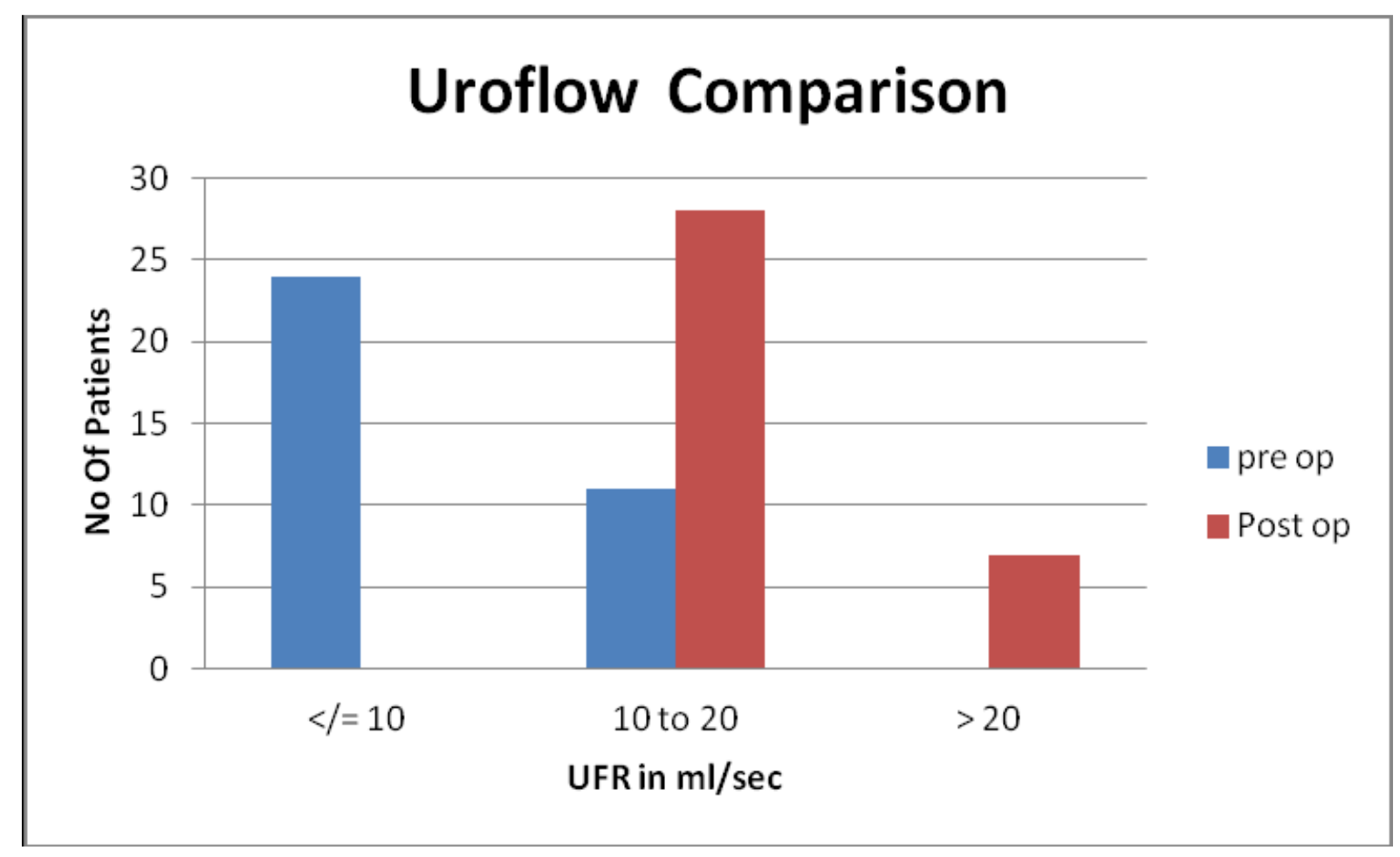

Fig-2: Comparison of pre and post-operative uroflowmetry results

33 patients underwent single-stage dorsal onlay buccal mucosa substitution urethroplasty and 2 patients underwent augmented roof strip buccal mucosa urethroplasty with operative time ranging from 3 hrs to 6 hrs with the mean operative time of $3.7 \mathrm{hrs}$.

Post-operatively 2 patients got surgical site infection and patients were given voiding trail after 4 to 6 weeks with a mean catheter period of 4.17 weeks.

Post-operatively 14 patients developed post void dribbling. Out of 35 patients 2 patients giving the history of erectile dysfunction. Donor site pain ranges from mild to moderate with good mouth opening.

Patients were followed up post-operatively at 3,6, 9, 12 and 36 months intervals out of 35 patients 6 patients had recurrence, out of 6 patients 4 patients underwent Visual Internal Urethrotomy at 6 and 12 months post-operative period out of these 4 patients 3 patients underwent one time Visual Internal Urethrotomy and 1 patient underwent Visual Internal Urethrotomy twice and considered as failure. Two patients presented with complete obstruction underwent complete lay open of urethra with perineal urethrostomy later underwent stage II urethroplasty and considered as failures. 


\section{Discussion}

BXO is a complex skin disease with unknown aetiology It was described as occurring in combination with an autoimmune disorder, genetic factors, infection and hormonal influence. Trauma, injury and sexual abuse have been suggested as possible triggers in genetically predisposed individuals [5]. LS has been reported as the most frequent cause of long segment urethral stricture, especially in India [6].

It involves prepuce, glans and anterior urethra and a challenge when considering urethral reconstruction that will have a minimal recurrence. Surgery is the treatment of choice in BXO related stricture urethra, though regression of the disease observed with topical application of steroids in few patients with acute stage of the disease. In a study by A Hartley et al, the effectiveness of medical therapy for the treatment of BXO show limited success. Many cases eventually required surgical treatmen [7].

Several substitution options for reconstruction of urethral strictures are used like genital skin, bladder mucosa and buccal mucosa. But in cases of BXO, the skin is involved or may become involved during the followup hence not recommended, as $90 \%$ recurrence noted in a study by I. Depasquale [8].

Buccal mucosa is hairless, and the tough epithelium makes it tough yet easy to handle. It also has a thin and highly vascular lamina propria, which facilitates inoculation and imbibition and thus tissue harvesting. The donor site heals quickly with minimal morbidity and complications. Hence, since 1998 BMG has become the choice for urethral augmentation or reconstruction. So now the trends have moved towards using single-stage repair for difficult strictures of anterior urethra due to $\mathrm{BXO}$, and the substitute of choice is BMG $[9,10]$.

In this study all cases underwent single-stage substitution even for long segment strictures as Francisco E. Martins et al shown in their review study, a 2-stage Johanson urethroplasty was not as successful as the buccal mucosal graft procedure, 64\% versus $82.5 \%$, respectively [6].

In this study results demonstrate that single stage dorsal on lay buccal mucosal urethroplasty provides satisfactory results in select cases of BXO related anterior urethral strictures. This study also indicates that, when the urethral plate is adequate, dorsal buccal mucosal grafting can be successfully used for reconstructing any urethral segment, including the meatus. Very few studies in literature shown buccal mucosa graft in BXO cases single stage procedure. Our approach is based on the technique of Kulkarni et al, and Deepak Dubey et.al., who presented their experience in patients with panurethral strictures related to BXO treated with 1-stage dorsal on lay buccal mucosal urethroplasty using a single perineal incision $[11,12]$.

Out of 35 patients 8 patients were underwent one or more procedures like dilatation, meatoplasty or visual internal urethrotomy before they underwent definitive urethral reconstructive surgery for stricture urethra. 9 patients were having meatal stenosis pre-operatively in whom buccal mucosa graft placed till meatus, all these patients voided well post-operatively. It suggests that even for meatal and fossa naviculars strictures buccal mucosa graft on-lay urethroplasty gives the good surgical outcome.

Similar results were observed in a study by Simsek et al where they performed circular buccal mucosal urethroplasty in 15 males for $\mathrm{BXO}$ related to anterior urethral strictures involving meatus, during subsequent visits, cosmetic outcome, symptoms assessment, and uroflowmetry over 20.5 months (range, 4-96 mo) were measured. The 15 men manifested no recurrent stricture, a normal meatus, and no chordee or erectile dysfunction. Excellent functional and cosmetic results were achieved in all 15 patients [13].

In this study age wise incidence of $\mathrm{BXO}$ showing the highest in 40 to 50 years age group and lowest in the extremes that is less than 10 years and more than 60 years age group. Patients who present early with stricture disease were having near normal serum creatinine values but who were presenting with chronic stricture disease having creatinine more than $2 \mathrm{mg} \%$.

Uroflowmetry done in all cases pre and postoperatively. with a mean pre- operative value as $9 \mathrm{ml} / \mathrm{sec}$ and mean post-operative value as $19.1 \mathrm{ml} / \mathrm{sec}$. On the statistical analysis of pre and post-operative uroflowmetry values, the ' $p$ ' value is 0.0001 (paired ' $t$ ' test) which is statistically significant. 
Meatal involvement in BXO related strictures is universal and the most accepted technique for reconstruction has been the ventral island fasciocutaneous flap, as described by Jordan [14] and Armenakas and McAninch [15]. In this study, the meatal component of the stricture was dealt simultaneously with proximal stricture repair. In patients in whom the urethral plate is severely stenosed, 2 cases in this study underwent augmented roof strip buccal mucosa urethroplasty.

Donor site was sutured for all patients. post-operatively 11 patients complained of moderate pain that is they required oral analgesics for pain control, rest of the patients had mild pain. mouth opening was good postoperatively. None of the patients had donor site wound infection.

Erectile dysfunction is one of the complications of urethral reconstructive surgeries, but incidence reduced with substitution urethroplasty. The incidence of impotence after urethral reconstruction ranges from $16.2 \%$ to $72 \%$. severe oedema and inflammation are responsible for erectile dysfunction after surgery. Oedema in the tissue surrounding the posterior urethra is more likely to impair the cavernous nerve fibres, potentially leading to the observed erectile dysfunction after surgery [16]. In this study $4(11.4 \%)$ patients required internal urethrotomy later out of 4 patients 1 patient underwent twice internal urethrotomy considered as failure. and 2 cases $(5.75 \%)$ underwent complete lay open of urethra after 6 months because of graft loss and severe urethral stenosis. Out of 35 patients 14 patients (40\%) complained of post-void dribbling, this is more when compared with Barbagli's study [17] which was $17 \%$ for dorsally placed grafts though other studies have shown ventral grafts causes more of post void dribbling.

Urethral strictures where the primary etiology other than BXO treated with buccal mucosa on lay graft as a single stage procedure shown similar results that is $82.8 \%, 87 \%$ and $90.6 \%$ success rate respectively in studies by G. barbagli et, al: Hussain Ahmad et, al : and Miodrag Acimovic et al, suggesting, irrespective of aetiology for anterior urethral stricture the results are good with buccal mucosa dorsal on-lay urethroplasty $[18,19,20]$. In a study by S.B Kulkarni et al, the overall success rate was $83.7 \%$ with a success rate of $86.5 \%$ for primary urethroplasty and $61.5 \%$ in patients in whom urethroplasty had previously failed. Most recurrent strictures developed at the proximal end of the graft [21].

Another study done by M.D. Mchembe et al in south Africa got similar results in their study with buccal mucosa graft dorsal on-lay procedure that is $90 \%$ success rate with single stage procedure [3].

Our study success rate is $91.42 \%$ which corroborates those of Kulkarni et.al. and Deepak Dubey et.al., who reported their experience with single stage buccal mucosa urethroplasty showed a success rate of $88 \%$ with the mean followup of 32.5 months [4]. Our study result suggestive of buccal mucosa graft on lay urethroplasty is one of the best options for BXO related urethral stricture.

\section{Conclusion}

For $\mathrm{BXO}$ related strictures, including meatal involvement,

a. Single stage substitution urethroplasty using buccal mucosa graft has excellent surgical outcome

b. These strictures are amenable for augmentation with buccal graft urethroplasty.

long-term follow-up is required to see the results of urethral stricture surgery.

Funding: Nil, Conflict of interest: None initiated, Permission from IRB: Yes

Ethical committee statement:- institute ethical committee approval taken for this study.

\section{Bibliography}

1. Das S. Susruta, the pioneer urologist of antiquity. J Urol. 2001 May;165(5):1405-8.

2. Tritschler S, Roosen A, Füllhase C, Stief CG, Rübben H. Urethral stricture: etiology, investigation and treatments. Dtsch Arztebl Int. 2013 Mar;110 (13): 220-6. doi: 10.3238/arztebl.2013.0220. Epub 2013 Mar 29.

3. M.D. Mchembe, A. Kategile, C.M.A. Yongolo, K.A. Mteta; Balanitis Xerotica Obliterans: An experience with Buccal Mucosa Onlay Flap Graft.;East and Central African Journal of Surgery, Vol. 16, No. 2, July/August, 2011, pp. 130-134.

4. Michael J. Belsante, J. Patrick Selph, Andrew C. Peterson; The contemporary management of urethral strictures in men resulting from lichen sclerosus; 
Translational Andrology and Urology,2015;4(1):22-28, doi: 10.3978/j.issn.2223-4683.2015.01.08.

5. Bjekić M, Šipetić S, Marinković J. Risk factors for genital lichen sclerosus in men. Br J Dermatol. 2011 Feb;164(2):325-9.doi:10.1111/j.1365-2133.2010. 10091.x.

6. Francisco E.Martins, Sanjay B. Kulkarni, Pankaj Joshi, JonathanWarner, and Natalia Martins; Management of Long-Segment and Panurethral Stricture Disease; Advances in Urology, Vol. 2015, Article ID 853914, 15 pages. http://dx.doi.org/10. 1155/ 2015/853914.

7. A Hartley, C. Ramanathan, H. Siddiqui, The surgical treatment of Balanitis Xerotica Obliterans; Indian Journal of Plastic Surgery January-April 2011 Vol 44 Issue 1; pg 91-97.

8. Depasquale I, Park AJ, Bracka A. The treatment of balanitis xerotica obliterans. BJU Int. 2000 Sep;86 (4):459-65.

9. Onkar Singh,Shilpi Singh Gupta,Nand Kishore Arvind,Anterior Urethral Strictures:A Brief Review of the CurrentSurgical Treatment. urologia internationalis; 2011; 86:1-10. doi: 10.1159/000319501.

10. Dubey D, Kumar A, Mandhani A, Srivastava A, Kapoor R, Bhandari M. Buccal mucosal urethroplasty: a versatile technique for all urethral segments. BJU Int. 2005 Mar;95(4):625-9.

11. Dubey D, Sehgal A, Srivastava A, Mandhani A, Kapoor R, Kumar A. Buccal mucosal urethroplasty for balanitis xerotica obliterans related urethral strictures: the outcome of 1 and 2-stage techniques. J Urol. 2005 Feb;173(2): 463-6.

12. S.B. Kulkarni, J.S. Kulkarni, D.V. Kirpekar,:A new technique for urethroplasty for balanitis xerotica obliterans,J Urol, 163 (suppl) (2000), p. 352.

13. Abdulmuttalip Simsek, Sinasi Yavuz Onol, Omer Kurt, Treatment of urethral strictures in balanitis xerotica obliterans (BXO) using circular buccal mucosal meatoplasy: Experience of 15 cases:Archivio Italiano di Urologia e Andrologia 2014; 86,1.doi: 10.4081/aiua.2014.1.23.

14. Jordan GH. Reconstruction of the fossa navicularis. J Urol. 1987 Jul;138(1):102-4.

15. Armenakas NA, McAninch JW. Management of fossa navicularis strictures. Urol Clin North Am. 2002 May; 29(2):477-84.

16. Hong Xie, Yue-Min Xu, Qiang Fu, Ying-Long Sa, Yong Qiao; The relationship between erectile function and complex panurethral stricture: a preliminary investigative and descriptive study; Asian Journal of Andrology (2015) 17, 315-318; doi: 10.4103/1008682X. 143312.

17. Marchal C, Pérez JE, Herrera B, Saez F, Castillo E, Antuña F, Julve E, Machuca FJ. Barbagli's dorsal urethroplasty. Analysis of results and factors for success. Arch Esp Urol. 2010 Sep;63(7):537-44.

18. Barbagli G, Guazzoni G, Lazzeri M. One-stage bulbar urethroplasty: retrospective analysis of the results in 375 patients. Eur Urol. 2008 Apr;53(4):82833. doi:10.1016/j.eururo.2008.01.041.Epub2008 Jan 22.

19. Hussain Ahmad, Arshad Mahmood, Waqar Azim Niaz, Muhammad Akmal, Badar Murtaza, Amir Nadim,: Bulbar urethral stricture repair with buccal mucosa graft urethroplasty, J Pak Med Assc 2011; 61 (5): 440-2.

20. Acimovic M, Milojevic B, Milosavljevic M, Skrodzka M, Radovanovic M, Rafailovic D, Dzamic Z, Djokic JH, Djinovic R. Primary dorsal buccal mucosa graft urethroplasty for anterior urethral strictures in patients with lichen sclerosus. Int Urol Nephrol. 2016 Apr; 48 (4):541-5. doi: 10.1007/s11255-015-1202-y. Epub 2016 Jan 11.

21. S. B. Kulkarni, P. M. Joshi, andK.Venkatesan, "Management of panurethral stricture disease in India," Journal of Urology,2012, vol.188, no. 3, pp. 824-830.

\section{How to cite this article?}

Manne V, Kulkarni P. Single stage substitution urethroplasty using buccal mucosa in the management of stricture urethra in balanitis xerotica obliterans (BXO). Int J Med Res Rev 2016;4(9):1675-1680.doi:10.17511/ijmrr. 2016.i09.27. 